OPEN ACCESS

Edited by:

Salvatore Galati,

Neurocenter of Southern

Switzerland, Switzerland

Reviewed by:

Davide Ferrazzoli,

Hospital of Vipiteno (SABES-ASDAA),

Daria Dinacci,

Clinica Hildebrand Centro di

Riabilitazione, Switzerland

*Correspondence:

Erwin E. H. van Wegen e.vanwegen@amsterdamumc.nl

Specialty section: This article was submitted to Movement Disorders, a section of the journal

Frontiers in Neurology

Received: 05 June 2020 Accepted: 21 August 2020

Published: 22 October 2020

Citation:

van Wegen EEH, Hirsch MA, van de Berg WDJ, Vriend C, Rietberg MB, Newman MA, Vanbellingen $T$ and van den Heuvel OA (2020) High-Intensity Interval Cycle Ergometer Training in

Parkinson's Disease: Protocol for Identifying Individual Response Patterns Using a Single-Subject Research Design.

Front. Neurol. 11:569880. doi: 10.3389/fneur.2020.569880

\title{
High-Intensity Interval Cycle Ergometer Training in Parkinson's Disease: Protocol for Identifying Individual Response Patterns Using a Single-Subject Research Design
}

\section{Erwin E. H. van Wegen ${ }^{1 *}$, Mark A. Hirsch ${ }^{2}$, Wilma D. J. van de Berg ${ }^{3}$, Chris Vriend ${ }^{3,4}$, Marc B. Rietberg ${ }^{5}$, Mark A. Newman ${ }^{2}$, Tim Vanbellingen ${ }^{6,7}$ and Odile A. van den Heuvel ${ }^{3,4}$ on behalf of the HIIT-PD Consortium}

1 Department of Rehabilitation Medicine, Amsterdam Movement Sciences, Amsterdam Neuroscience, Amsterdam University Medical Center (UMC), Vrije Universiteit Amsterdam, Amsterdam, Netherlands, ${ }^{2}$ Department of Physical Medicine and Rehabilitation, Carolinas Medical Center, Carolinas Rehabilitation, Charlotte, NC, United States, ${ }^{3}$ Department of Anatomy \& Neurosciences, Amsterdam Neuroscience, Amsterdam University Medical Center (UMC), Vrije Universiteit Amsterdam, Amsterdam, Netherlands, ${ }^{4}$ Department of Psychiatry, Amsterdam Neuroscience, Amsterdam University Medical Center (UMC), Vrije Universiteit Amsterdam, Amsterdam, Netherlands, ${ }^{5}$ Department of Rehabilitation Medicines, Amsterdam Movement Sciences, Amsterdam University Medical Center (UMC), Vrije Universiteit Amsterdam, Amsterdam, Netherlands, ${ }^{6}$ Neurocenter, Luzerner Kantonsspital, Lucerne, Switzerland, ${ }^{7}$ Gerontechnology and Rehabilitation Group, University of Bern, Bern, Switzerland

Background: People with Parkinson's disease (PD) experience not only motor problems but also non-motor problems that seriously impede their daily functioning and quality of life. The current pharmacologic treatment of PD is symptomatic, and alternative rehabilitation treatments, which preferably also have a disease-modifying effect and promote neuroplasticity, are needed. Recent studies suggest that high-intensity interval training $(\mathrm{HIIT})$ is promising for promoting neuroplasticity in human PD, with short training time and reduced burden. Biomarkers for neuroplasticity such as brain-derived neurotrophic factor (BDNF) and neurodegeneration (including neurofilament NfL and $\alpha$-synuclein) may play a role, but their response to HIIT is not well-investigated.

Objectives: The aims of this study were (1) to study the effects of 4 weeks of HIIT compared with 4 weeks of continuous aerobic exercise on motor and non-motor outcomes of PD and (2) to investigate the association between HIIT, motor/non-motor performances changes, and blood biomarker levels for neuroplasticity and neurodegeneration.

Study Design: Single-subject research design with alternating treatment setup (ABACA) and frequent repeated measurements was used. Each participant received different intervention conditions $(B / C)$ interspersed with baseline periods (A, i.e., ABACA or ACABA), and frequent repeated assessment of outcome measures is done to quantify within-subject, individual response patterns with sufficient power for data analysis. Blood samples were collected once a week in the baseline and training phases (A1 and B/C) and once every 2 weeks in the washout phases (A2 and $A 3$ ). 
Intervention: Four subjects with PD on stable dopaminergic medication, two in HoehnYahr stage 1-2, and two in Hoehn-Yahr stage 2.5-3 followed an ABACA or ACABA schedule, consisting of blocks with 30-min sessions of "B" (HIIT) or 50-min sessions of "C" [continuous aerobic exercise (CAE)] 3x/week for 4 weeks, separated by baseline "A" periods of 8 weeks for a total duration of 28 weeks.

Outcome Measures: Outcome measures include disease status [Movement Disorder Society-sponsored revision of the Unified Parkinson's Disease Rating Scale (MDSUPDRS)], blood biomarkers (BDNF, Nfl, and $\alpha$-synuclein), measures for functional mobility (including an activity tracker), and activities of daily living, as well as cognition, mood, biorhythm (sleeping problems), and quality of life.

Data Analysis: Visual analysis of trends in level, slope, and variability in response patterns was carried out, confirmed by longitudinal regression analysis with phase (ABACA) as the independent variable.

Keywords: Parkinson's disease, high intensity (strenuous) exercise, neuroplastic changes, BDNF, NFL, synuclein alpha

\section{INTRODUCTION}

Parkinson's disease (PD) is a complex, chronic, highly disabling progressive neurodegenerative movement disorder, characterized by the loss of dopamine neurons in the substantia nigra (1). The disease is characterized by motor (e.g., tremor, gait disturbance, bradykinesia, and falls) and also nonmotor (e.g., depression, reduced cognitive performance, and biorhythm/sleep) symptoms (2). To date, there is no cure. Current treatment paradigms are mainly aimed at alleviating motor and psychiatric features but do not consider individual differences in treatment response or molecular profile. Moreover, several motor (e.g., gait disturbances and balance disturbances) and non-motor (e.g., mood disturbances, cognitive functioning, and sleep problems) features respond insufficiently to current pharmacological treatments; and unfortunately, we do not have rehabilitative strategies that halt or slow down disease progression $(2,3)$. Frequent physical exercise training is increasingly recognized as an effective therapy for PD and other neurodegenerative diseases that may even exert diseasemodifying influences through neuroplastic mechanisms $(4,5)$.

Aerobic and/or progressive resistance exercise based on the American College of Sports Medicine recommendation of $3 \times /$ week, $30-60 \mathrm{~min} / \mathrm{session}$ for at least 12 weeks demonstrate long-term ( $>12$ weeks) improvements in both motor performance [e.g., walking speed, aerobic capacity, and lower-limb muscle strength; (6)] and cognitive performance (attention and executive functioning) in PD patients $(3,7)$. Shorter-lasting aerobic exercise programs $(3 \times /$ week, 30-60 $\mathrm{min} / \mathrm{session}$ for 4 weeks) may also show improvements in motor performance (e.g., walking speed and aerobic capacity), though the long-term effects of short-term aerobic exercise are less consistent (4).

Physical training studies show promising results in terms of exercise-induced neuroplasticity, at both symptom and molecular levels in human and animal models of exercise and PD (8). For example, voluntary treadmill running in mice for $40 \mathrm{~min} /$ day, 5 days/week for 18 weeks improved motor coordination and aerobic capacity and reduced nigrostriatal neuronal loss (9). The improvements were associated with increased levels of BDNF in the substantia nigra and striatum. A recent review and a meta-analysis showed that HIIT has acute and long-term effects on serum BDNF levels $(10,11)$. Several studies suggest that serum BDNF concentration levels are reduced in PD patients [for example, Frazzitta et al. (12)]. Low BDNF is associated not only with cognitive impairments in PD patients (13) but also with mood disturbances (14). Biomarkers for neuroplasticity, such as BDNF, and biomarkers for (synaptic) neurodegeneration (including neurofilament NfL and $\alpha$-synuclein) are, respectively, decreased and increased in PD patients compared with age-matched controls (15-17). Studies suggest intensive physical training to be promising for modulating these biomarkers in people with $\mathrm{PD}$ (11, 12). BDNF has shown to respond well to intensive physical training; for serum NfL and $\alpha$-synuclein, this has not yet been properly investigated.

A promising, time-efficient, and highly effective physical exercise strategy is "high-intensity interval training" (HIIT), which only recently is being explored in clinical research (18). HIIT encompasses several intervals of short-lasting, highintensity bouts alternated with low-intensity bouts, that has shown to match or even surpass the cardiovascular responses to moderate continuous aerobic exercise (CAE) (19). This increased efficiency is achieved in a shorter period of total training time, which may enhance the motivation of PD patients and help promote adopting a more active lifestyle (19). For example, HIIT for three 40-min sessions per week for 8 weeks has been suggested to evoke enhanced adaptive aerobic capacity (e.g., increased aerobic capacity) as well as increased cognitive performance, as compared with CAE in healthy older adults and older adults with mild cognitive impairment (19). Similar results have been 
found for adult type 2 diabetes mellitus patients (18) and adult cardiovascular patients (4).

Only a few studies to date have investigated the effect of HIIT on motor and non-motor outcomes in PD. High feasibility of HIIT in PD was recently demonstrated (20): participants were able to be complete $45 \mathrm{~min}$ of HIIT three times a week for 12 weeks without intervention-related dropouts and $>80 \%$ compliance. Group session HIIT consisted of an exercise gym circuit designed to elicit a physiological response indicative of high-intensity exercise ( $\geq 85 \%$ HRmax). Fernandes et al. (21) performed a pilot randomized controlled single-blind trial to study the effect of 12 weeks of HIIT vs. moderateintensity continuous exercise (MICE) training in PD. They enrolled 20 participants, with Hoehn-Yahr stage 1-3, who performed walking/jogging training three times per week for 12 weeks. The HIIT protocol consisted of a 1-min walking/jogging bout at rating of perceived exertion (Borg scale RPE) scale 15-17 level alternated with 2 min of walking at 9-11 level of RPE during a 25-min session. MICE training consisted of $26 \mathrm{~min}$ of walking/jogging at 11-14 level of RPE. HIIT improved 6-min walk test distance and increased endothelial reactivity (a marker for increased blood flow). These values did not change with MICE. They did not measure nonmotor outcomes. O'Callaghan et al. (22) entered the sample by Harvey et al. into a new comparison in a 12 -week pilot study of HIIT or MICE. Their study supports the feasibility of HIIT in PD and found that HIIT increased circulating serum brain-derived neurotrophic factor (BDNF), a protein that may play a key role in neuroplasticity maintaining or improving brain functions (10). BDNF levels were higher after HIIT compared with continuous moderate-intensity aerobic training (22).

The combined evidence of animal and human studies suggests that motor performance, cognitive performance, and mood in PD are related and that biomarkers for neuroplasticity and neurodegeneration are associated with both motor and non-motor features. This underpins the importance of disease-modifying treatment in PD including aerobic and/or progressive resistance exercise as adjuncts to pharmacological treatment for the control of motor and non-motor features. As such, determining the optimal type and dosage of exercise interventions to obtain these neuroplastic effects is an important focus of current neurorehabilitation research in $\operatorname{PD}(8,23)$.

Researchers argue that the heterogeneity of patient populations and use of classic intervention study design are major confounding factors in making progress toward determining optimal type and dosage of exercise interventions (24). Within-participant research designs with frequently repeated measurements (i.e., single case experimental designs) may be more suitable than group studies to capture individual recovery patterns. This design allows for more continuous repeated measures of the target outcomes, at baseline, during, and after the intervention period (25). Incorporation of many repeated measures allows a detailed analysis of individual responses with adequate power. No single study has compared the effects of HIIT with CAE on changes in motor performance, cognitive abilities, mood disturbances, and biorhythms/sleep in people with PD and whether improvements are associated with quantitative changes in blood-based biomarkers (BDNF, NfL, and $\alpha$-synuclein).

Therefore, the first objective of this pilot study is to explore the response patterns of motor and non-motor performance and serum biomarker levels to 4 weeks of HIIT compared with 4 weeks of traditional moderate CAE in four single subjects with PD. The second objective is to explore the associations between changes in motor and non-motor outcome measures and serum neuroplasticity and neurodegeneration biomarkers for both exercise conditions.

Our central hypothesis is that HIIT is more effective at improving motor performance, cognition, mood, and sleep as well as increasing concentration of BDNF and decreasing NfL and $\alpha$-synuclein levels than moderate-intensity CAE. In addition, we expect that HIIT-induced changes in serum BDNF, NfL, and $\alpha$-synuclein protein levels are associated with changes in motor and non-motor performance of PD patients.

\section{METHODS}

\section{Study Design}

We will use a single-subject alternating treatment approach with A1-B-A2-C-A3 or A1-C-A2-B-A3 design to study the effects of HIIT on motor and non-motor performance and neuroplasticity and neurodegeneration biomarkers such that subjects will perform both the HIIT (B-phase) and the moderate CAE training (C-phase) for 4 weeks. After a baseline (A1) period of 4 weeks, the first intervention period of 4 weeks will take place (B or C), followed by a washout period of 8 weeks without an intervention (A2), the second intervention period of 4 weeks (B or C), and finally a third washout period of 8 weeks (A3). Each patient therefore experiences the full set of treatment approaches. The order of interventions $B$ and $C$ will be randomized for each participant (i.e., ABACA or ACABA, two of each, four participants total) using a random sequence generator. Assessment will be done by Good Clinical Practice (GCP)-trained blinded assessors.

\section{Study Population}

This exploratory pilot study aims to recruit a convenience sample of four subjects with confirmed idiopathic PD on stable dopaminergic medication via the outpatient clinic for movement disorders at Amsterdam UMC, Vrije Universiteit Medical Center, Amsterdam, the Netherlands. Patients are asked in the study information letter to maintain a stable medication schedule throughout the study, unless the treating neurologist deems it medically necessary to adapt drug treatment. When applicable, this will be recorded and taken into account in the analysis. The study was approved by the Medical Ethics Committee of Amsterdam UMC, Vrije Universiteit Medical Center (nr. 2029.083). The study will be conducted in compliance with the Declaration of Helsinki. 


\section{Inclusion and Exclusion Criteria}

Inclusion criteria are (1) diagnosis of idiopathic PD according to UK Brain Bank criteria (26); (2) Hoehn-Yahr stage 1 ( $n=2$ patients) and $2.5-3(n=2$ patients) as confirmed by a trained assessor; (3) age between 55 and 80 years; (4) sufficient cognition to comprehend training instruction [Montreal Cognitive Assessment (MoCA) score > 21]; and (5) able to provide written informed consent. Exclusion criteria are (1) history of neurologic deficits other than PD; (2) severe fluctuating responses to dopaminergic medication; (3) psychiatric, musculoskeletal, or metabolic disorders prohibiting participation in intensive exercise training; (4) cardiovascular disorders or cardiac risk prohibiting participation in intensive exercise training as assessed by Lausanne Questionnaire [ $>2$ risk items scored "yes"; Bille et al. (27)]; and (5) participation in a professionally supervised high-intensity therapy/exercise program to improve fitness in the 2 months before inclusion.

\section{Measurement Procedures and Training}

All testing and training will be performed in the "on" state, $\sim 1-1.5 \mathrm{~h}$ after medication intake. Prior to allocation, subjects will complete baseline testing for demographics and for motor and non-motor symptoms (Table 1). Assessment of demographics and baseline characteristics will include, age, height, weight, sex, disease duration, Movement Disorder Society-sponsored revision of the Unified Parkinson's Disease Rating Scale (MDS-UPDRS I to IV), Hoehn-Yahr stage and LEDD (levodopa equivalent daily dose), medication schedule and usage (dopaminergic, psychopharmacological, and any other medications), and number of falls in past month.

\section{$\mathrm{VO}_{2}$-Max Test Procedure}

At the end of the $\mathrm{A} 1$ phase, subjects will perform a $\mathrm{VO}_{2}$ Max test to determine individual training load, as described by Mavromatti et al. (28). In brief, the exercise test will be conducted on an electronically braked cycle ergometer (Excalibur Sport, Lode, the Netherlands), integrated with a cardiopulmonarymonitoring system that controls the work rate protocol on the ergometer and records breath-by-breath measurements of $\mathrm{VO}_{2}$, $\mathrm{VCO}_{2}$, ventilation, and heart rate (Cosmed Benelux BV, the Netherlands) throughout the test. Handlebars and saddle of the cycle ergometer will be adjusted to match each participant's anthropometrics. The work rate protocol consists of 2-min steps starting with unloaded cycling and then increasing to $50 \mathrm{~W}$, and thereafter increasing in steps of $25 \mathrm{~W}$. While the ergometer maintains a constant workload, independent of cadence, participants are instructed to aim for cadence of $\sim 60-$ $80 \mathrm{rpm}$. At the end of each step, participants are asked to rate their level of exertion (rating of perceive exertion) using the Borg RPE 10-point category ratio scale (Borg CR10 scale, "0" to "10") (29). Participants are verbally encouraged to carry on for as long as possible. The exercise test is terminated by voluntary exhaustion, by a cadence $<45 \mathrm{rpm}$ or for safety reasons, compliant with the American College of Sports Medicine's guidelines for clinical exercise testing (30). Subjects are asked to abstain from exercise and consumption of alcohol or caffeine $12 \mathrm{~h}$ prior to testing.

\section{Outcome Measures}

Outcome measures are chosen based on recommendations by the Movement Disorders Society (31), the 2014 European guideline of physical therapy for PD (32), and recommendations by the members of the project team, including several PD patients. Table 1 shows the timeline and assessment schedule for demographics and all outcome parameters. Demographics include age, sex, disease duration, Hoehn-Yahr stage, medication schedule and dosage, and MoCA score.

The primary study parameter is disease severity measured every 4 weeks during baseline A1 and A2 and intervention and two times during baseline A3 with the MDS-UPDRS part III (motor examination) during on-state (33).

Secondary outcome parameters were pre-post intervention outcome measures will be administered before and after each study phase; and disease status with MDS-UPDRS 1, II, and IV (33). Cognition will be evaluated with the MoCA (34), the Stroop test (35), and the Trail Making Test for executive function [TMT; Olchik et al. (36)] and the PD Cognitive Functioning Rating Scale (PD-CFRS) (37). Activities of daily living and quality of life were assessed with selfreport questionnaires: the Nottingham Extended ADL index (NEAI) (38) and the Parkinson Disease Questionnaire (PDQ8) (39). Mood and sleep will be assessed using the Beck Depression Inventory questionnaire (31) and the Scopa Sleep scale (40), respectively.

Weekly outcomes are motor performance, namely, the 10meter walk test (41), the Timed "Up-and-Go" test (TUG) (42), and the one-leg stance test (43). In addition, daily physical activity with a wrist-worn activity tracker will be recorded throughout the study. For weekly self-report outcomes, we will use Ecological Momentary Assessment methodology (44), asking frequent (weekly in baseline phases, three times a week in intervention phases), short online survey questions about nonmotor functions using Castor EDC (45): Mood will be assessed with a visual analog scale (VAS) score rating: "Can you rate your current mood on a scale from "1" to "10"?" (a score of " 1 " represents "very sad"; a score of "10" represents "very happy"). Sleep performance will be assessed with a VAS score rating: "Can you rate how well you slept last night on a scale from " 1 " to " 10 "?" (A score of "1" represents "very bad"; a score of "10" represents "very good"). Cognitive function will be assessed with a VAS score for "ability to concentrate": "Can you rate well you are able to concentrate on daily tasks on a scale from " 1 " to "10"?" (a score of "1" represents "very bad"; a score of "10" represents "very good"). In addition, we will ask the subjects to keep a logbook of their attended sessions and perceived effort (RPE).

Blood samples (for serum BDNF, NfL, and $\alpha$-synuclein) will be collected by a trained nurse once a week in the baseline and training phases $(\mathrm{A} 1$ and $\mathrm{B} / \mathrm{C})$. Blood draw frequency is decreased to once every 2 weeks in the washout phases (A2 and A3) to reduce patient burden. Twenty-milliliter blood samples will be taken from the antecubital vein at rest in the morning hours between 08:00 and 10:00 in the fasting state. We will use anticoagulant-free tubes, which be placed on ice $\left(-80^{\circ} \mathrm{C}\right)$ until further analysis. BDNF concentration will be measured with a 
TABLE 1 | Assessment schedule for outcomes across the 28-week study period.

\section{Schedule}

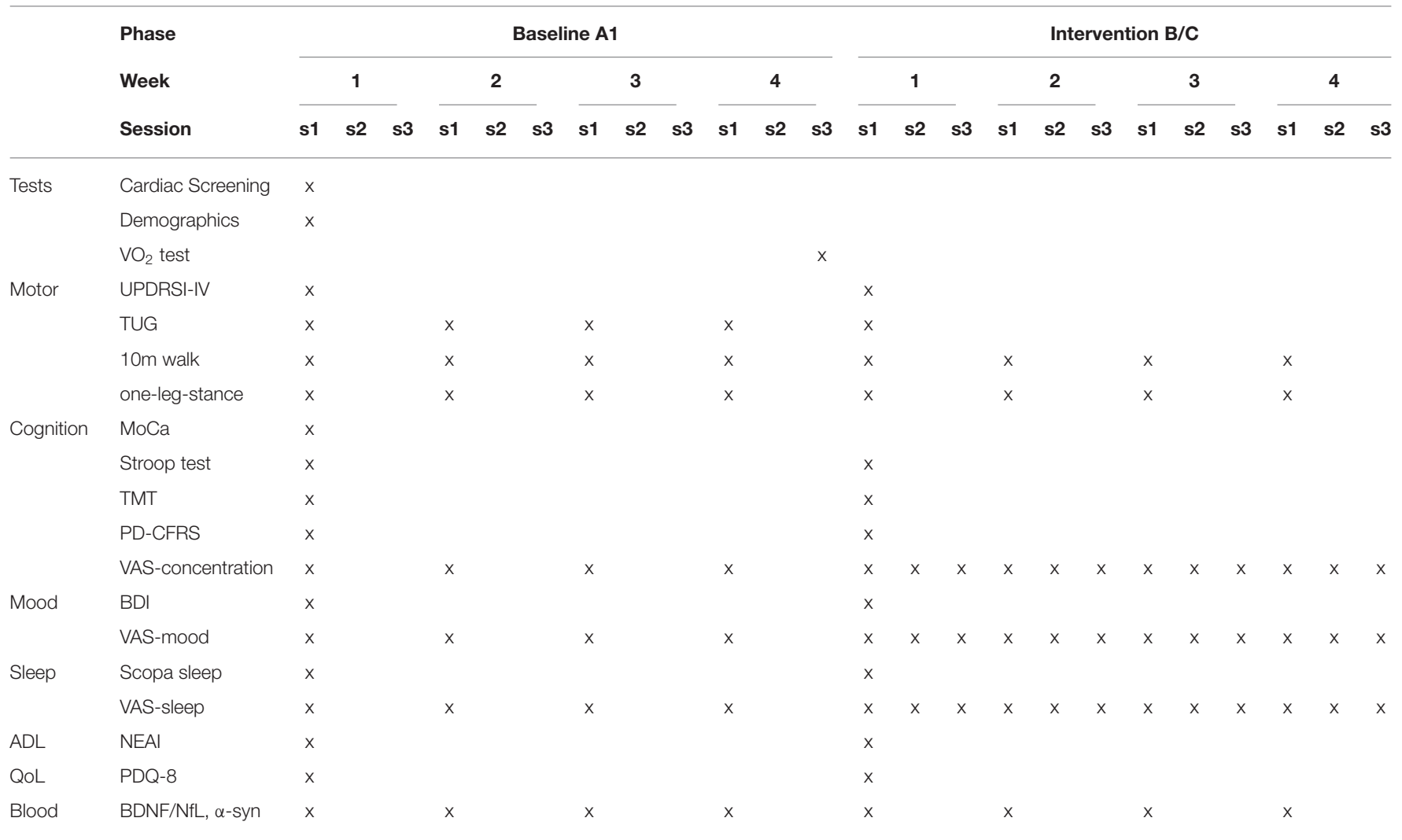

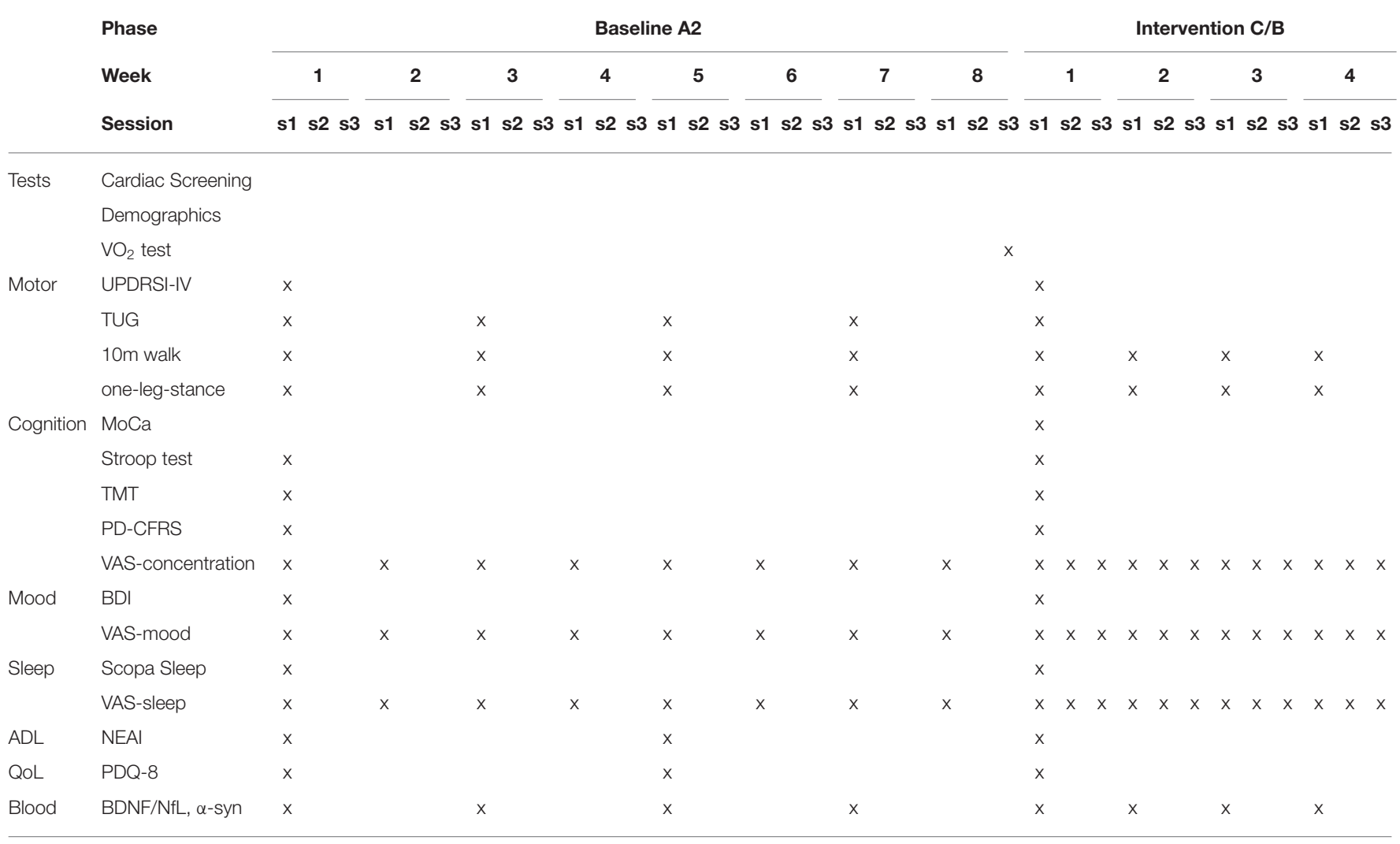


TABLE 1 | Continued

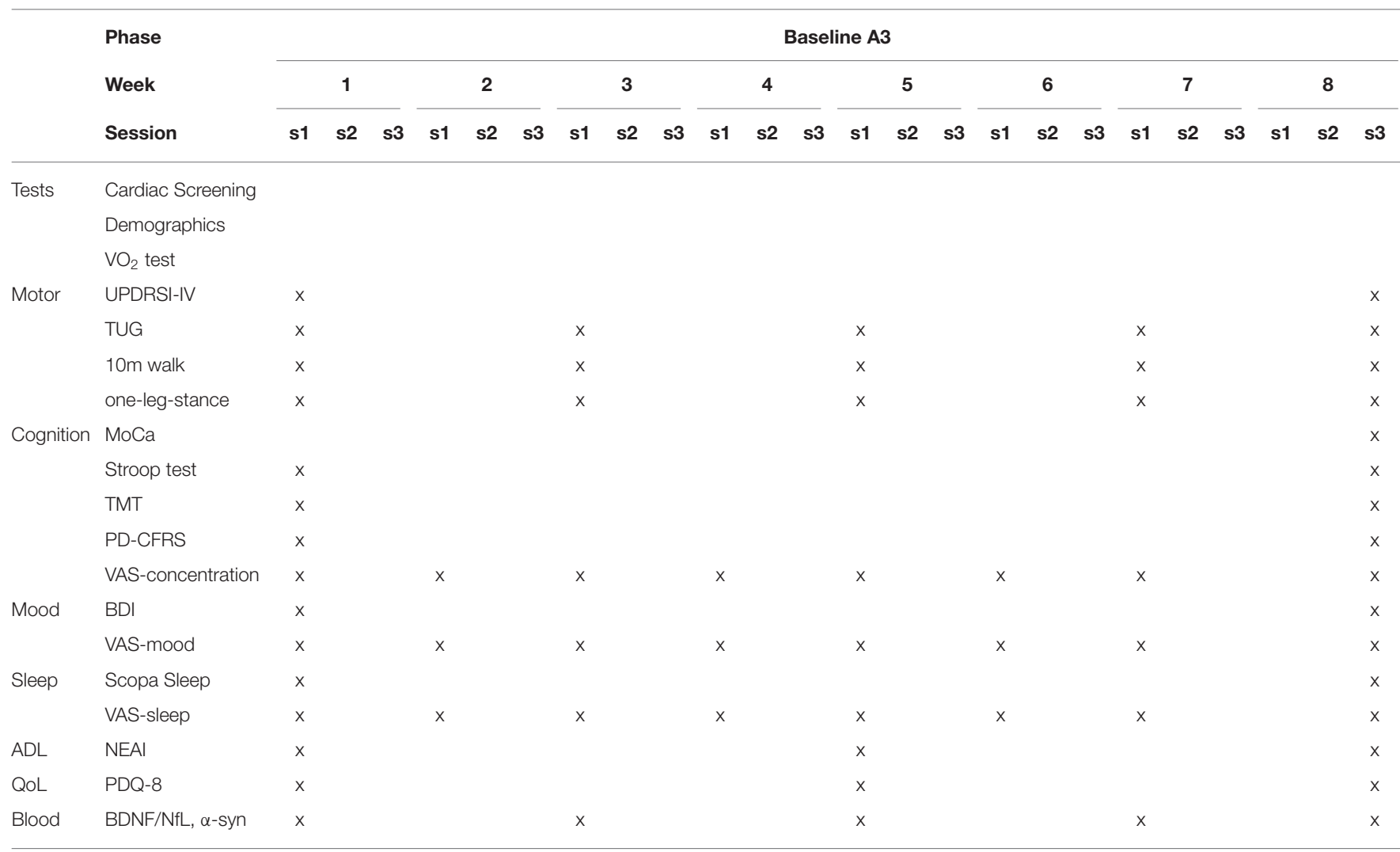

Planning of baseline (A1, A2, and A3), intervention (B and C), and measurements (crosses indicate time movements). Pre-post phase measurements will be performed before and after both baseline and both intervention phases.

UPDRS, MDS-Unified Parkinson's Disease Rating Scale; MoCa, Montreal Cognitive Assessment; PD-CFRS, PD Cognitive Functioning Rating Scale; Stroop, Stroop test; VAS, visual analog scale; BDI, Beck Depression Inventory; ADL, activities of daily living; Scopa Sleep, Sleep scale from Scopa; NEAl, Nottingham Extended ADL index; QoL, quality of Life; PDQ-8, Parkinson's Disease Questionnaire; BDNF, brain-derived neurotrophic factor; NfL, neurofilament light; $\alpha$-syn, alpha synuclein.

commercially available and in-house validated kit (Quanterix Simoa). Serum NfL and $\alpha$-synuclein concentrations will be measured using an in-house developed Homebrew Simoa assay $(17,46)$.

\section{Training Protocol}

Subjects will perform HIIT or moderate CAE on a stationary bicycle (Lode Corival, Lode Inc.) $3 \times /$ week for two periods of 4 weeks in a group of four subjects (Figure 1). The order of the intervention phases will be randomized. Training will take place on Mondays, Wednesdays, and Fridays, to allow for sufficient recovery periods between training sessions.

Each HIIT session is about 30 min of interval training, starting with a 5 min of warm-up at $25-35 \% \mathrm{~W}$-max. Subsequently, six to eight blocks of HIIT will be performed, alternating $45 \mathrm{~s}$ at $>85 \% \mathrm{VO}_{2}$-max, with $90 \mathrm{~s}$ at $30-40 \% \mathrm{~W}$-max. After the interval block, 5 min of cooling-down will be performed at $20-35 \% \mathrm{~W}$ max. Workload will be progressively adapted using a fixed weekly schedule or upon therapist decision by adding intervals at the end of the sessions.
Each CAE session is about $50 \mathrm{~min}$ of continuous training at a preset workload of about 55\% W-max. Workload will be progressively adapted after every week by reducing rest time, based on a fixed schedule or upon therapist decision. A trained physiotherapist-supervisor, assisted by GCP-trained interns from the Faculty of Human Movement Sciences will assist the subjects with water, music, and motivational encouragements. Throughout the study, patients are asked to maintain their regular medication.

The Ecological Momentary Assessments taken weekly (A phases) or every other day (B/C phases) using the online survey throughout the study period will act automatically as reminders and will help with compliance to training and assessments. We will comply with consensus recommendations of Slade et al. (47) regarding reporting on exercise interventions.

\section{Statistical Analysis}

Although the protocol specifies primary and secondary study outcomes, we will assess the results based on a combination of those parameters.

For example, the primary research parameter that we have specified is the MDS-UPDRS-III, which is measured before and 

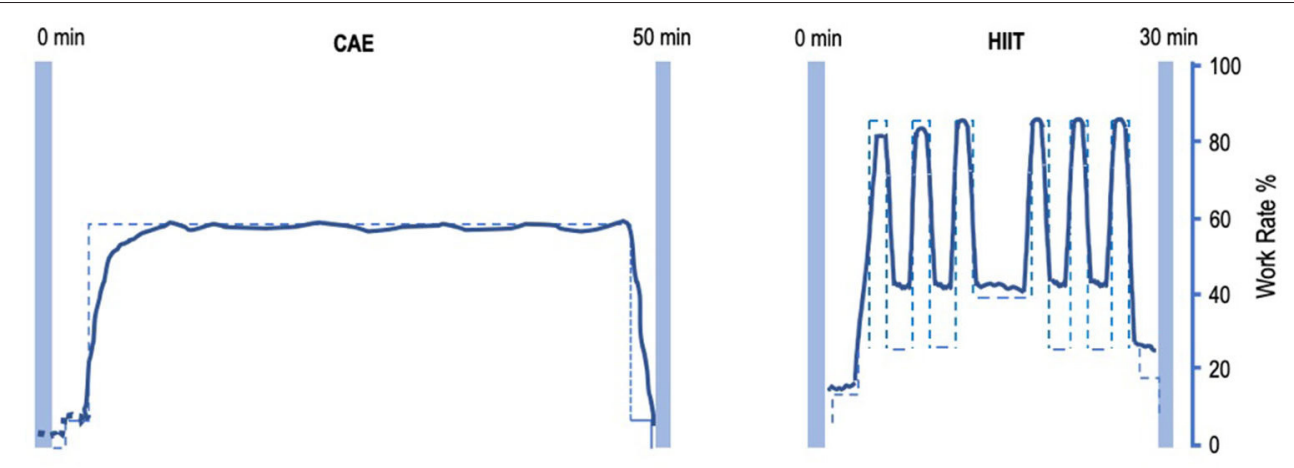

FIGURE 1 | Intensity-work rate plot for CAE and HIIT session. Left: intensity-work rate plot for a 50-min CAE intervention session. Right: intensity-work rate plot for a 30-min HIIT intervention session. CAE training intensity will be at about 55\% W-max, HIIT will alternate between training intensity $35 \%$ W-max during low-intensity intervals and $>85 \%$ W-max at high-intensity intervals. CAE, continuous aerobic exercise; HIIT, high-intensity interval training.

after each phase. If, under the influence of the interventions, there are improvements within the participants that are greater than the measurement error of 3.25 points known from the literature (48), this is a clear indication that the intervention influences motor function. However, if there is less or no change in the MDS-UPDRS-III but in one or more other parameters, this is still informative with regard to the effects of the different interventions and the underlying mechanisms. If no change is visible in any parameter under the influence of the interventions in any participant (through visual analysis and/or statistical tests), then the conclusion should be that there is no effect.

Visual inspection is the method of choice for single-subject research designs (SSRDs) (24). We will inspect, analyze, and describe the changes across the baseline and intervention phases (phase mean level and data spread), and we will compare the trend (slope) of scores with the baseline mean levels and variability (standard deviations) on outcomes for each intervention strategy and each patient. Longitudinal regression analysis with "phase" (i.e., condition) as the independent variable will be used for further confirmation $(\alpha=0.05)$.

We will not explicitly test for group differences between patients in Hoehn-Yahr stage 1-2 or for patients in Hoehn-Yahr stage 2.5-3, but we will get insights on the direction of effects and effect sizes in the intervention phases compared with the baseline phases, which we will report descriptively for both participants in both subgroups.

The association between biomarkers and symptom changes will be quantified by Pearson correlation coefficients and corrected for age, between changes in BDNF, $\alpha$ synuclein, and $\mathrm{Nfl}$ and change scores on primary and secondary parameters.

\section{DISCUSSION}

Accumulating scientific evidence supports the use of physical rehabilitation training and exercise as an effective nonpharmacologic therapy to improve motor symptoms, problems with mobility and gait (49, 50), and non-motor features, including mood, cognition, and sleep [for review, see Reynolds et al. (51)] for patients at all stages of PD. Intensive exercise may even induce neuroplastic disease-attenuating effects via endogenous production of neurotrophic factors, including $\operatorname{BDNF}(4,5,52,53)$.

There has been considerable progress in the area of physical training-induced neuroplasticity effects in $\operatorname{PD}(11,54,55)$, though our understanding is far from complete. Studies reported that PD patient show increased serum NfL protein levels (17) compared to age-matched healthy controls. NfL is a marker for axonal damage and related to cortical degeneration in PD (15). As we have stated earlier, the effect of physical exercise training on NfL serum protein levels in early or late stage PD is currently unknown.

Neurotrophic factors, including BDNF, are soluble endogenously produced polypeptides that are involved in the development, growth, functioning, and regulation of neurons and neuron-supporting cells. Studies indicate that continuous high-intensity training, moderate-intensity CAE, or low-intensity exercise enhances serum and plasma BDNF levels $(12,22,52,56)$. A recent meta-analysis showed cumulative evidence of physical exercise induced improved serum BDNF protein levels in PD (11). It is plausible that besides activation of the neurotrophic pathway (10), physical training increases blood flux into basal ganglia (57) and that this increased flux may stimulate vascular endothelial cells to secrete BDNF as a response to blood shear stress in the vessels (58). BDNF also has known cytoprotective effects on the striatum (59).

Reduced aerobic and anaerobic capacity are common in the elderly and especially in $\mathrm{PD}$ patients, though the rate of capacity reduction can be decreased by adopting a more active physical lifestyle (1). The experimental intervention in this study, HIIT, has already been used in sports science to improve both aerobic and anaerobic capacity (18); and preliminary studies are emerging in the field of $\mathrm{PD}$. It is plausible that HIIT can be a time-efficient type of exercise that integrates both aerobic and anaerobic exercise, thereby enhancing the motivation of PD patients to comply with the intervention and in the long run promote a more active 
lifestyle. To our knowledge, we are the first to explore the within-subjects responses of blood biomarkers with weekly repeated measurements over multiple weeks (during baseline, washout, and intervention periods) for the two different exercise conditions. The design of the study allows for careful individual exploration of the response patterns of different exercise strategies on both symptoms and biomarkers. The advantage is that a small sample is enough to get important new knowledge, which will facilitate future well-powered clinical trials.

This study primarily explores whether HIIT is more effective at improving motor and cognitive performance than moderate CAE. If our hypothesis that HIIT is more effective than moderate CAE is correct, future HIIT studies in PD patients can add other treatment types, such as cognitive stimulation. For example, the Park-in-Shape study (60) investigated the feasibility of adding "exergaming" elements to improve adherence. Furthermore, a recent publication of a large observational study in PD patients in the Netherlands highlights the cost-effectiveness of specialized goal-based physical activity therapy by reducing both health-care cost and disease-related complications (61), which should be explored in future studies by combining HIIT with exercise goals beyond mere completion of the protocol.

To increase the societal relevance and impact of the current study protocol, two Parkinson patients who were consulted during the design process of this study expressed optimism about the procedures presented in this protocol. They also acknowledged the need for a better understanding of individual neural response patterns to different exercise intensities.

\section{DATA AVAILABILITY STATEMENT}

The original contributions presented in the study are included in the article/supplementary material, further inquiries can be directed to the corresponding author/s.

\section{ETHICS STATEMENT}

The studies involving human participants were reviewed and approved by Medical Ethics Committee of Amsterdam UMC, Vrije Universiteit Medical Center (nr. 2029.083). The participants provided their written informed consent.

\section{REFERENCES}

1. Garcia-Ruiz PJ, Espay AJ. Parkinson disease: an evolutionary perspective. Front Neurol. (2017) 8:157. doi: 10.3389/fneur.2017.00157

2. Titova N, Chaudhuri KR. Non-motor Parkinson disease: new concepts and personalised management. Med J Aust. (2018) 208:404-9. doi: 10.5694/mja17.00993

3. Mak MK, Wong-Yu IS, Shen X, Chung CL. Long-term effects of exercise and physical therapy in people with Parkinson disease. Nat Rev Neurol. (2017) 13:689-703. doi: 10.1038/nrneurol.2017.128

4. Pedersen BK, Saltin B. Exercise as medicine-evidence for prescribing exercise as therapy in 26 different chronic diseases. Scand J Med Sci Sports. (2015) 25:1-72. doi: $10.1111 /$ sms. 12581

\section{AUTHOR CONTRIBUTIONS}

EW, MH, WB, TV, MR, MN, and $\mathrm{OH}$ contributed to conception and design of the study. EW and $\mathrm{MH}$ wrote the first draft of the manuscript. EW, MH, OH, TV, and WB wrote sections of the manuscript. All authors contributed to manuscript revision, read, and approved the submitted version. On behalf of the HIITPD consortium members who contributed to topical discussions during the grant writing proces: H. W. Berendse, Y. D. van der Werf, D. Ferrazzoli, R. T. Jaspers, G. Kwakkel, V. de Groot.

\section{FUNDING}

This work was funded by a research grant from the Parkinson Vereniging Netherlands (2019-R05), The Dutch Brain Foundation (DR-2019-00311) and a planning grant from Amsterdam Movement Sciences (2018) awarded to EW.

\section{ACKNOWLEDGMENTS}

We thank Ruud Overes and Sara Riggare for their input as a patient expert.

\section{CONSORTIUM}

H. W. Berendse ${ }^{1}$, Y. D. van der Werf ${ }^{2}$, D. Ferrazzoli ${ }^{3}$, R. T. Jaspers ${ }^{4}$, G. Kwakkel ${ }^{5}$, Y. Mahieu ${ }^{5}$, E. Goudzwaard ${ }^{5}$ and V. de Groot ${ }^{5}$

${ }^{1}$ Department of Neurology, Amsterdam Neuroscience, Amsterdam University Medical Center (UMC), Vrije Universiteit Amsterdam, Amsterdam, Netherlands

${ }^{2}$ Department of Anatomy \& Neurosciences, Amsterdam Neuroscience, Amsterdam University Medical Center (UMC), Vrije Universiteit Amsterdam, Amsterdam, Netherlands

${ }^{3}$ Department of Parkinson's disease, Movement Disorders \& Brain Injury Rehabilitation, Fresco Parkinson Center, 'MoriggiaPelascini' Hospital - Gravedona ed Uniti, Como, Italy

${ }^{4}$ Laboratory for Myology, Department of Human Movement Sciences, Amsterdam Movement Sciences, Vrije Universiteit Amsterdam, Amsterdam, Netherlands

${ }^{5}$ Department of Rehabilitation Medicine, Amsterdam Movement Sciences, Amsterdam Neuroscience, Amsterdam University Medical Center (UMC), Vrije Universiteit Amsterdam, Amsterdam, Netherlands.

5. Ferreira RN, de Miranda AS, Rocha NP, Simoes E, Silva AC, Teixeira AL, et al. Neurotrophic factors in Parkinson's disease: what have we learned from pre-clinical and clinical studies? Curr Med Chem. (2018) 25:3682-702. doi: 10.2174/0929867325666180313101536

6. Hirsch MA, Toole T, Maitland CG, Rider R. The Effects of balance training and high-intensity resistance training on persons with idiopathic Parkinson's disease. Arch Phys Med Rehabil. (2003) 84:1109-17. doi: 10.1016/S0003-9993(03)00046-7

7. van Wegen EEH, Hirsch MA, Huiskamp M, Kwakkel G. Harnessing cueing training for neuroplasticity in Parkinson disease. Top Geriatr Rehabil. (2014) 30:46-57. doi: 10.1097/TGR.0000000000000005

8. Petzinger GM, Fisher BE, McEwen S, Beeler JA, Walsh JP, Jakowec MW. Exercise-enhanced neuroplasticity targeting motor and cognitive 
circuitry in Parkinson's disease. Lancet Neurol. (2013) 12:716-26. doi: 10.1016/S1474-4422(13)70123-6

9. Lau YS, Patki G, Das-Panja K, Wei-Dong L, Ahmad SO. Neuroprotective effects and mechanisms of exercise in a chronic mouse model of Parkinson's disease with moderate neurodegeneration. Euro J Neurosci. (2011) 33:126474. doi: $10.1111 / \mathrm{j} .1460-9568.2011 .07626 . x$

10. Jimenez-Maldonado A, Renteria I, Garcia-Suarez PC, Moncada-Jiminez J, Freire-Royes LF. The impact of high-intensity interval training on brain derived neurotrophic factor in brain: a mini-review. Front Neurosci. (2018) 12:839. doi: $10.3389 /$ fnins.2018.00839

11. Hirsch MA, van Wegen EEH, Newman MA, Heyn PC. Exercise-induced increase in brain-derived neurotrophic factor in human Parkinson's disease: a systematic review and meta-analysis. Transl Neurodegener. (2018) 7:7. doi: 10.1186/s40035-018-0112-1

12. Frazzitta G, Maestri F, Ghilardi MF, Riboldazzi G, Perini M, Bertotti $\mathrm{G}$, et al. Intensive rehabilitation increases BDNF serum levels in parkinsonian patients: an RCT. Neurorehabil Neural Repair. (2014) 28:163-8. doi: $10.1177 / 1545968313508474$

13. Wang Y, Liu H, Zhang BS, Soares JC, Zhang XY. Low BDNF is associated with cognitive impairments in patients with Parkinson's disease. Parkinsonism Relat Disord. (2016) 29:66-71. doi: 10.1016/j.parkreldis.2016.05.023

14. Wang $\mathrm{Y}$, Liu $\mathrm{H}, \mathrm{Du} \mathrm{XD}$, Zhang $\mathrm{Y}$, Yin G, Zhang BS, et al. Association of low serum BDNF with depression in patients with Parkinson's disease. Parkinsonism Relat Disord. (2017) 41:73-8. doi: 10.1016/j.parkreldis.2017.05.012

15. Leaver K, Poston KL. Do CSF biomarkers predict progression to cognitive impairment in Parkinson's disease patients? A systematic review. Neuropsychol Rev. (2015) 25:411-23. doi: 10.1007/s11065-015-9307-8

16. Scalzo P, Kümmer A, Bretas TL, Cardoso F, Teixeira AL. Serum levels of brainderived neurotrophic factor correlate with motor impairment in Parkinson's disease. J Neurol. (2010) 257:540-5. doi: 10.1007/s00415-009-5357-2

17. Oosterveld LP, Verberk IMW, Majbour NK, El-Agnaf OM, Weinstein HC, Berendse HW, et al. CSF or serum neurofilament light added to $\alpha$-Synuclein panel discriminates Parkinson's from controls. Mov Disord. (2020) 35:288-95. doi: $10.1002 / \mathrm{mds} .27897$

18. Tjønna AE, Lee SJ, Rognmo $\varnothing$, Stølen TO, Bye A, Haram PM, et al. Aerobic interval training versus continuous moderate exercise as a treatment for the metabolic syndrome. Circulation. (2008) 118:346-54. doi: 10.1161/CIRCULATIONAHA.108.772822

19. Enette L, Vogel T, Fanon JL, Lang PO. Effect of interval and continuous aerobic training on basal serum and plasma brain-derived neurotrophic factor values in seniors: a systematic review of intervention studies. Rejuvenation Res. (2017) 20:473-83. doi: 10.1089/rej.2016.1886

20. Harvey M, Weston KL, Gray WK, O'Callaghan A, Oates LL, Davidson R, et al. High-intensity interval training in people with Parkinson's disease: a randomized, controlled feasibility trial. Clin Rehabil. (2018) 33:428-38. doi: $10.1177 / 0269215518815221$

21. Fernandes B, Barbieri FA, Arthuso FZ, Silva FA, Moretto GF, Imaizumi LFI, et al. High-intensity interval versus moderate-intensity continuous training in individuals with Parkinson's disease: hemodynamic and functional adaptation. J Phys Act Health. (2020) 17:85-91. doi: 10.1123/jpah.2018-0588

22. O'Callaghan A, Harvey M, Houghton D, Gray WK, Weston KL, Oates LL, et al. Comparing the influence of exercise intensity on brain-derived neurotrophic factor serum levels in people with Parkinson's disease: a pilot study. Aging Clin Exp Res. (2020) 32:1731-8. doi: 10.1007/s40520-019-01353-w

23. Abbruzzese G, Marchese R, Avanzino L, Pelosin E. Rehabilitation for Parkinson's disease: current outlook and future challenges. Parkinsonism Related Disord. (2016) 22:S60-4. doi: 10.1016/j.parkreldis.2015.09.005

24. Krasny-Pacini A, Evans J. Single-case experimental designs to assess intervention effectiveness in rehabilitation: a practical guide. Ann Phys Rehabil Med. (2018) 61:164-79. doi: 10.1016/j.rehab.2017.12.002

25. Ledford JR, Gast DL. Single Subject Research Methodology in Behavioral Sciences. New York, NY: Routledge Press (2009).

26. Hughes AJ, Daniel SE, Kilford L, Lees AJ. Accuracy of clinical diagnosis of idiopathic Parkinson's disease: a clinico-pathological study of 100 cases. J Neurol Neurosurg Psychiatry. (1992) 55:181-4. doi: 10.1136/jnnp.55. 3.181
27. Bille K, Figueiras D, Schamasch P, Kappenberger L, Brenner JI, Meijiboom FJ, et al. Sudden cardiac death in athletes: the lausanne recommendations. Eur J Cardiovasc Prev Rehabil. (2006) 13:859-75. doi: 10.1097/01.hjr.0000238397.50341.4a

28. Mavrommati F, Collett J, Franssen M, Meaney A, Sexton C, DennisWest A, et al. Exercise response in Parkinson's disease: insights from a cross-sectional comparison with sedentary controls and a per-protocol analysis of a randomised controlled trial. BMJ Open. (2017) 7:e017194. doi: 10.1136/bmjopen-2017-017194

29. Borg GA. Psychophysical bases of perceived exertion. Med Sci Sports Exerc. (1982) 14:377-81. doi: 10.1249/00005768-198205000-00012

30. ACSM's. ACSM Guidelines for Exercise Testing and Prescription. 9th ed. Pescatello LS, Arena R, Riebe D, Thompson PD, editors. Philadelphia, PA: Wolters Kluwer/Lippincott Williams \& Wilkins (2014). p. 456.

31. Schrag A, Barone P, Brown RG, Leentjens AF, McDonald WM, Starkstein $S$, et al. Depression rating scales in Parkinson's disease: critique and recommendations. Mov Disord. (2007) 27:1077-92. doi: 10.1002/mds.21333

32. Keus SHJ, Munneke M, Graziano M, Paltamaa J, Pelosin E, Domingos J, et al. European Physiotherapy Guideline for Parkinson's Disease. Amersfoort: KNGF/ParkinsonNet (2014).

33. Goetz CG, Tilley BC, Shaftman SR, Stebbins GT, Fahn S, Martinez-Martin P, et al. Movement Disorder Society UPDRS Revision Task Force. Movement disorder society-sponsored revision of the unified Parkinson's disease rating scale (MDS-UPDRS): scale presentation and clinimetric testing results. Mov Disord. (2008) 23:2129-70. doi: 10.1002/mds.22340

34. Hoops S, Nazem S, Siderowf AD, Duda JE, Xie SX, Stern MB, et al. Validity of the MoCA and MMSE in the detection of MCI and dementia in Parkinson disease. Neurology. (2009) 73:1738-45. doi: 10.1212/WNL.0b013e3181c34b47

35. Sisco SM, Slonena E, Okun MS, Bowers D, Price CC. Parkinson's disease and the Stroop color word test: processing speed and interference algorithms. Clin Neuropsychol. (2016) 30:1104-17. doi: 10.1080/13854046.2016.1188989

36. Olchik MR, Ghisi M, Freiry AM, Ayres A, Schuh AFS, Rieder CR, et al. Comparison trail making test between individuals with Parkinson's disease and health controls: suggestions of cutoff point. Psychol Neurosci. (2017) 10:77-82. doi: 10.1037/pne0000076

37. Ruzafa-Valiente E, Fernández-Bobadilla R, García-Sánchez C, Pagonabarraga J, Martínez-Horta S, Kulisevsky J. Parkinson's disease-cognitive functional rating scale across different conditions and degrees of cognitive impairment. $J$ Neurol Sci. (2016) 361:66-71. doi: 10.1016/j.jns.2015.12.018

38. Clarke CE, Patel S, Ives N, Rick CE, Dowling F, Woolley R, et al. Physiotherapy and occupational therapy vs no therapy in mild to moderate Parkinson disease: a randomized clinical trial. JAMA Neurol. (2016) 73:291-9. doi: 10.1001/jamaneurol.2015.4452

39. Luo N, Tan LC, Zhao Y, Lau PN, Au WL, Li SC. Determination of the longitudinal validity and minimally important difference of the 8-item Parkinson's disease questionnaire (PDQ-8). Mov Disord. (2009) 24:183-7. doi: $10.1002 / \mathrm{mds} .22240$

40. Högl B, Arnulf I, Comella C, Ferreira J, Iranzo A, Tilley B, et al. Scales to assess sleep impairment in Parkinson's disease: critique and recommendations. Mov Disord. (2010) 25:2704-16. doi: 10.1002/mds.23190

41. Lang JT, Kassan TO, Devaney LL, Colon-Semenza C, Joseph MF. Testretest reliability and minimal detectable change for the 10-meter walk test in older adults with Parkinson's disease. J Geriatr Phys Ther. (2016) 39:165-70. doi: 10.1519/JPT.0000000000000068

42. Huang SL, Hsieh CL, Wu RM, Tai CH, Lin CH, Lu WS. Minimal detectable change of the timed "up \& go" test and the dynamic gait index in people with Parkinson disease. Phys Ther. (2011) 91:114-21. doi: 10.2522/ptj.20090126

43. Prime M, McKay JL, Bay AA, Hart AR, Kim C, Abraham A, et al. Differentiating parkinson disease subtypes using clinical balance measures. $J$ Neurol Phys Ther. (2020) 44:34-41. doi: 10.1097/NPT.0000000000000297

44. Maher JP, Rebar AL, Dunton GF. Ecological momentary assessment is a feasible and valid methodological tool to measure older adults' physical activity and sedentary behavior. Front Psychol. (2018) 9:1485. doi: $10.3389 /$ fpsyg. 2018.01485

45. Castor EDC. Castor Electronic Data Capture. (2020). Available online at: https://castoredc.com

46. Kuhle J, Barro C, Andreasson U, Derfuss T, Lindberg R, Sandelius $A$, et al. Comparison of three analytical platforms for quantification 
of the neurofilament light chain in blood samples: ELISA, electrochemiluminescence immunoassay and Simoa. Clin. Chem. Lab. Med. (2016) 54:1655-61. doi: 10.1515/cclm-2015-1195

47. Slade SC, Dionne CE, Underwood M, Buchbinder R, Beck B, Bennell K, et al. Consensus on Exercise reporting template (CERT): modified delphi study. Phys Ther. (2016) 96:1514-24. doi: 10.2522/ptj.20150668

48. Horváth K, Aschermann Z, Ács P, Deli G, Janszky J, Komoly S, et al. Minimal clinically important difference on the Motor Examination part of MDS-UPDRS. Parkinsonism Relat Disord. (2015) 21:1421-6. doi: 10.1016/j.parkreldis.2015.10.006

49. Koop MM, Rosenfeldt AB, Alberts JL. Mobility improves after high intensity aerobic exercise in individuals with Parkinson's disease. J Neurol Sci. (2019) 399:187-93. doi: 10.1016/j.jns.2019.02.031

50. Uygur M, Bellumori M, Knight CA. Effects of low-resistance, interval bicycling intervention in Parkinson's disease. Physiother Theory Pract. (2017) 33:897-904. doi: 10.1080/09593985.2017.1359868

51. Reynolds GO, Otto MW, Ellis TD, Cronin-Golomb A. The therapeutic potential of exercise to improve mood, cognition, and sleep in Parkinson's disease. Mov Disord. (2016) 31:23-38. doi: 10.1002/mds.26484

52. Landers MR, Navalta JW, Murtishaw AS, Kinney JW, Richardson SP. A high-intensity exercise boot camp for persons with Parkinson's disease: a phase II, pragmatic, randomized clinical trial of feasibility, safety, signal of efficacy, and disease mechanisms. J Neurol Phys Ther. (2019) 43:12-25. doi: 10.1097/NPT.0000000000000249

53. Karssemeijer EGA, Aaronson JA, Bossers WJR, Donders R, Olde Rikkert MGM, Kessels RPC. The quest for synergy between physical exercise and cognitive stimulation via exergaming in people with dementia: a randomized controlled trial. Alz Res Therapy. (2019) 11:3. doi: 10.1186/s13195018-0454-z

54. Sacheli MA, Neva JL, Lakhani B, Murray DK, Vafai N, Shahinfard E, et al. Exercise increases caudate dopamine release and entral striatal activation in Parkinson's disease. Mov Disord. (2019) 34:1891-900. doi: 10.1002/mds.27865

55. Hirsch MA, Farley BG. Exercise, neuroplasticity and Parkinson's disease. Eur J Phys Rehabil Med. (2009) 45:215-29.
56. Morberg BM, Jensen J, Bode M, Wermuth L. The impact of high intensity physical training on motor and non-motor symptoms in patients with Parkinson's disease (PIP): a preliminary study. NeuroRehabilitation. (2014) 35:291-8. doi: 10.3233/NRE-141119

57. Alberts JL, Linder SM, Penko AL, Lowe MJ, Phillips M. It is not about the bike; it is about the pedaling: forced exercise and Parkinson's disease. Exerc Sport Sci Rev. (2011) 39:177-86. doi: 10.1097/JES.0b013e31822cc71a

58. Prigent-Tessier A, Quirié A, Maguin-Gaté K, Szostak J, Mossiat C, Nappey $\mathrm{M}$, et al. Physical training and hypertension have opposite effects on endothelial brain-derived neurotrophic factor expression. Cardiovasc Res. (2013) 100:374-82. doi: 10.1093/cvr/cvt219

59. Baydyuk M, Xu B. BDNF signaling and survival of striatal neurons. Front Cell Neurosci. (2014) 8:254. doi: 10.3389/fncel.2014.00254

60. van der Kolk NM, Overeem S, de Vries NM, Kessels RP, Donders R, Brouwer $\mathrm{M}$, et al. Design of the park-in-shape study: a phase II double blind randomized controlled trial evaluating the effects of exercise on motor and non-motor symptoms in Parkinson's disease. BMC Neurol. (2015) 15:56. doi: 10.1186/s12883-015-0312-6

61. Ypinga JH, de Vries NM, Boonen LHHM, Koolman X, Munneke M, Zwinderman $\mathrm{AH}$, et al. Effectiveness and costs of specialised physiotherapy given via ParkinsonNet: a retrospective analysis of medical claims data. Lancet Neurol. (2018) 17:153-61. doi: 10.1016/S1474-4422(17)30406-4

Conflict of Interest: The authors declare that the research was conducted in the absence of any commercial or financial relationships that could be construed as a potential conflict of interest.

Copyright (c) 2020 van Wegen, Hirsch, van de Berg, Vriend, Rietberg, Newman, Vanbellingen and van den Heuvel. This is an open-access article distributed under the terms of the Creative Commons Attribution License (CC BY). The use, distribution or reproduction in other forums is permitted, provided the original author(s) and the copyright owner(s) are credited and that the original publication in this journal is cited, in accordance with accepted academic practice. No use, distribution or reproduction is permitted which does not comply with these terms. 